\title{
System of Operator Quasi Equilibrium Problems
}

\author{
Suhel Ahmad Khan \\ Department of Mathematics, BITS-Pilani, Dubai Campus, P.O. Box 345055, Dubai, UAE \\ Correspondence should be addressed to Suhel Ahmad Khan; khan.math@gmail.com \\ Received 24 January 2014; Accepted 4 June 2014; Published 19 June 2014 \\ Academic Editor: Sivaguru Sritharan
}

Copyright (c) 2014 Suhel Ahmad Khan. This is an open access article distributed under the Creative Commons Attribution License, which permits unrestricted use, distribution, and reproduction in any medium, provided the original work is properly cited.

We consider a system of operator quasi equilibrium problems and system of generalized quasi operator equilibrium problems in topological vector spaces. Using a maximal element theorem for a family of set-valued mappings as basic tool, we derive some existence theorems for solutions to these problems with and without involving $\Phi$-condensing mappings.

\section{Introduction}

In 2002, Domokos and Kolumbán [1] gave an interesting interpretation of variational inequality and vector variational inequalities (for short, VVI) in Banach space settings in terms of variational inequalities with operator solutions (for short, OVVI). The notion and viewpoint of OVVI due to Domokos and Kolumbán [1] look new and interesting even though it has a limitation in application to VVI. Recently, Kazmi and Raouf [2] introduced the operator equilibrium problem which generalizes the notion of OVVI to operator vector equilibrium problems (for short, OVEP) using the operator solution. They derived some existence theorems of solution of OVEP with pseudomonotonicity, without pseudomonotonicity, and with $B$-pseudomonotonicity. However, they dealt with only the single-valued case of the bioperator. It is very natural and useful to extend a single-valued case to a corresponding setvalued one from both theoretical and practical points of view.

The system of vector equilibrium problems and the system of vector quasi equilibrium problems were introduced and studied by Ansari et al. [3, 4]. Inspired by above cited work, in this paper, we consider a system of operator quasi equilibrium problems (for short, SOQEP) in topological vector spaces. Using a maximal element theorem for a family of set-valued mappings according to [5] as basic tool, we derive some existence theorems for solutions to SOQEP with and without involving $\Phi$-condensing mappings.

Further, we consider a system of generalized quasi operator equilibrium problems (for short, SGQOEP) in topological vector spaces and give some of its special cases and derive some existence theorems for solutions to SOQEP with and without involving $\Phi$-condensing mappings by using well-known maximal element theorem [5] for a family of set-valued mappings, and, consequently, we also get some existence theorems for solutions to a system of operator equilibrium problems.

\section{Preliminaries}

Let $I$ be an index set, for each $i \in I$, and let $X_{i}$ be a Hausdorff topological vector space. We denote $L\left(X_{i}, Y_{i}\right)$, the space of all continuous linear operators from $X_{i}$ into $Y_{i}$, where $Y_{i}$ is topological vector space for each $i \in I$. Consider a family of nonempty convex subsets $\left\{K_{i}\right\}_{i \in I}$ with $K_{i}$ in $L\left(X_{i}, Y_{i}\right)$.

Let

$$
\begin{aligned}
& X=\prod_{i \in I} X_{i}, \\
& K=\prod_{i \in I} K_{i} .
\end{aligned}
$$

Let $C_{i}: K \rightarrow 2^{Y_{i}}$ be a set-valued mapping such that, for each $f \in K, C_{i}(f)$ is solid, open, and convex cone such that $0 \notin C_{i}(f)$ and $P_{i}=\bigcap_{f \in K} C_{i}(f)$.

For each $i \in I$, let $F_{i}: K \times K_{i} \rightarrow Y_{i}$ be a bifunction and let $A_{i}: K \rightarrow 2^{K_{i}}$ be a set-valued mapping with nonempty values. We consider the following system of operator quasi 
equilibrium problems (for short, SOQEP). Find $\bar{f} \in K$ such that, for each $i \in I$,

$$
\bar{f}_{i} \in A_{i}(\bar{f}), \quad F_{i}\left(\bar{f}, g_{i}\right) \notin-C_{i}(\bar{f}), \quad \forall g_{i} \in A_{i}(\bar{f}) .
$$

We remarked that, for the suitable choices of $I, F_{i}, K_{i}$, $X_{i}, Y_{i}, C_{i}$, and $A_{i}$, SOQEP (2) reduces to the problems considered and studied by [3-6] and the references therein.

Now, we will give the following concepts and results which are used in the sequel.

Definition 1. Let $M$ be a nonempty and convex subset of a topological vector space, and let $Z$ be a topological vector space with a closed and convex cone $P$ with apex at the origin. A vector-valued function $\phi: M \rightarrow Z$ is said to be as follows:

(i) P-function if and only if $\forall x, y \in M$ and $\lambda \in[0,1]$ :

$$
\phi(\lambda x+(1-\lambda) y) \in \lambda \phi(x)+(1-\lambda) \phi(y)-P
$$

(ii) natural P-quasifunction if and only if $\forall x, y \in M$ and $\lambda \in[0,1]:$

$$
\phi(\lambda x+(1-\lambda) y) \in \operatorname{Co}\{\phi(x), \phi(y)\}-P,
$$

where $C o B$ denotes the convex hull of $B$;

(iii) $P$-quasifunction if and only if $\forall \alpha \in Z$ and the set $\{x \in$ $M: \phi(x)-\alpha \in-P\}$ is convex.

Definition 2 (see [7]). Let $X$ be a topological vector space and let $L$ be a lattice with a minimal element, denoted by 0 . A mapping $\phi: 2^{X} \rightarrow L$ is called a measure of noncompactness provided that the following conditions hold for any $M, N \in$ $2^{X}$ :

(i) $\phi(\bar{C} o M)=\phi(M)$, where $\bar{C} o M$ denotes the closed convex hull of $M$;

(ii) $\phi(M)=o$ if and only if $M$ is precompact;

(iii) $\phi(M \cup N)=\max \{\phi(M), \phi(N)\}$.

Definition 3 (see [7]). Let $X$ be a topological vector space, $D \subset X$, and let $\phi$ be a measure of noncompactness on $X$. A set-valued mapping $T: D \rightarrow 2^{X}$ is called $\phi$-condensing provided that $M \subset D$ with $\phi(T(M)) \geq \phi(M)$; then $M$ is relative compact; that is, $\bar{M}$ is compact.

Remark 4. Note that every set-valued mapping defined on a compact set is $\phi$-condensing for any measure of noncompactness $\phi$. If $X$ is locally convex, then a compact set-valued mapping (i.e., $T(D)$ is precompact) is $\phi$-condensing for any measure of noncompactness $\phi$. Obviously, if $T: D \rightarrow 2^{X}$ is $\phi$-condensing and $T^{\prime}: D \rightarrow 2^{X}$ satisfies $T^{\prime}(x) \subset T(x)$, for all $x \in X$, then $T^{\prime}$ is also $\phi$-condensing.

The following maximal element theorems will play key role in establishing existence results.

Theorem 5 (see [8]). For each $i \in I$, let $K_{i}$ be a nonempty convex subset of a topological vector space $X_{i}$ and let $S_{i}, T_{i}$ : $K \rightarrow 2^{K_{i}}$ be the two set-valued mappings. For each $i \in I$, assume that the following conditions hold: (a) for all $x \in K, \operatorname{CoS}_{i}(x) \subseteq T_{i}(x)$;

(b) for all $x \in K, x_{i} \notin T_{i}(x)$;

(c) for all $y_{i} \in K_{i}, S_{i}^{-1}\left(y_{i}\right)$ is compactly open $K$;

(d) there exist a nonempty compact subset $D$ of $K$ and a nonempty compact convex subset $E_{i} \subseteq K_{i}$, for each $i \in$ $I$, such that, for all $x \in K \backslash D$, there exists $i \in I$ such that $S_{i}(x) \cap E_{i} \neq \emptyset$.

Then, there exists $\bar{x} \in K$ such that $S_{i}(\bar{x})=\emptyset$ for each $i \in I$.

We will use the following particular form of a maximal element theorem for a family of set-valued mappings due to Deguire et al. [5].

Theorem 6 (see [5]). Let I be any index set, for each $i \in I$, let $K_{i}$ be a nonempty convex subset of a Hausdorff topological vector space $X_{i}$, and let $S_{i}: K=\prod_{i \in I} K_{i} \rightarrow 2^{K_{i}}$ be a set-valued mapping. Assume that the following conditions hold:

(i) $\forall i \in I$ and $\forall x \in K$; $S_{i}(x)$ is convex;

(ii) $\forall i \in I$ and $\forall x \in K ; x_{i} \notin S_{i}(x)$, where $x_{i}$ is the ith component of $x$;

(iii) $\forall i \in I$ and $\forall y_{i} \in K_{i}$; $S_{i}^{-1}\left(y_{i}\right)$ is open $K$;

(iv) there exist a nonempty compact subset $D$ of $K$ and $a$ nonempty compact convex subset $E_{i} \subseteq K_{i}, \forall i \in I$ such that $\forall x \in K \backslash D$ and there exists $i \in I$ such that $S_{i}(x) \cap$ $E_{i} \neq \emptyset$.

Then, there exists $\bar{x} \in K$ such that $S_{i}(\bar{x})=\emptyset$ for each $i \in I$.

Remark 7. If $\forall i \in I, K_{i}$ is nonempty, closed, and convex subset of a locally convex Hausdorff topological vector space $X_{i}$, then condition (iv) of Theorem 6 can be replaced by the following condition:

$\left(\right.$ iv) ${ }^{1}$ the set-valued mapping $S: K \rightarrow 2^{K}$ is defined as $S(x)=\prod_{i \in I} S_{i}(x), \forall x \in K, \phi$-condensing.

\section{Main Result}

Throughout this paper, unless otherwise stated, for any index set $I$ and for each $i \in I$, let $Y_{i}$ be a topological vector space and let $K=\prod_{i \in I} K_{i}, C_{i}: K \rightarrow 2^{Y_{i}}$ be a set-valued mapping such that, for each $f \in K, C_{i}(f)$ is proper, solid, open, and convex cone such that $0 \notin C_{i}(f)$ and $P_{i}=\bigcap_{f \in K} C_{i}(f)$. We denote $L\left(X_{i}, Y_{i}\right)$, the space of all continuous linear operators from $X_{i}$ into $Y_{i}$. We also assume that $\forall i \in I, A_{i}: K \rightarrow 2^{K_{i}}$ is a set-valued mapping such that $\forall f \in K, A_{i}(f)$ is nonempty and convex, $A^{-1}\left(g_{i}\right)$ is open in $K, f_{i} \in K_{i}$, and the set $F_{i}:\{f \in$ $\left.K: f_{i} \in A_{i}(f)\right\}$ is closed in $K$, where $f_{i}$ is the $i$ th component of $f$. (2).

Now, we have the following existence result for SOQEP

Theorem 8. For each $i \in I$, let $K_{i}$ be nonempty and convex subset of a Hausdorff topological vector space $X_{i}$ and let $F_{i}: K \times$ $K_{i} \rightarrow Y_{i}$ be a bifunction. Suppose that the following conditions hold: 
(i) $\forall i \in I$ and $\forall f \in K, F_{i}\left(f, f_{i}\right) \notin-C_{i}(f)$, where $f_{i}$ is the ith component of $f$;

(ii) $\forall i \in I$ and $\forall f \in K$; the vector-valued function $g_{i} \mapsto$ $F_{i}\left(f, g_{i}\right)$ is natural $P_{i}$-quasifunction;

(iii) $\forall i \in I$ and $\forall g_{i} \in K_{i}$; the set $\left\{f \in K: F_{i}\left(f, g_{i}\right) \notin\right.$ $\left.-C_{i}(f)\right\}$ is closed in $K$;

(iv) there exist a nonempty compact subset $N$ of $K$ and a nonempty compact convex subset $B_{i}$ of $K_{i}$, for each $i \in I$ such that $\forall f \in K \backslash N$; there exists $i \in I$ and $\widehat{g}_{i} \in B_{i}$ such that $\widehat{g}_{i} \in A_{i}(f)$ and $F_{i}\left(f, \widehat{g}_{i}\right) \in-C_{i}(f)$.

Then SOQEP (2) has a solution.

Proof. Let us define, for each given $i \in I$, a set-valued mapping $T_{i}: K \rightarrow 2^{K_{i}}$ by

$$
T_{i}(f)=\left\{g_{i} \in K_{i}: F_{i}\left(f, g_{i}\right) \in-C_{i}(f)\right\}, \quad \forall f \in K .
$$

First, we claim that $\forall i \in I$ and $f \in K, T_{i}(f)$ is convex. Fix an arbitrary $i \in I$ and $f \in K$. Let $g_{i, 1}, g_{i, 2} \in T_{i}(f)$ and $\lambda \in[0,1]$; then we have

$$
F_{i}\left(f, g_{i_{j}}\right) \in-C_{i}(f), \quad \text { for } j=1,2 .
$$

Since $F_{i}(f, \cdot)$ is natural $P_{i}$-quasifunction, there exists $\mu \in[0,1]$ such that

$$
\begin{aligned}
F_{i}\left(f, \lambda g_{i, 1}+(1-\lambda) g_{i, 2}\right) \in & \mu F_{i}\left(f, g_{i, 1}\right) \\
& +(1-\mu) F_{i}\left(f, g_{i, 2}\right)-P_{i} .
\end{aligned}
$$

From the inclusion of $(i)$ and (ii), we get

$$
F_{i}\left(f, \lambda g_{i, 1}+(1-\lambda) g_{i, 2}\right) \in-C_{i}(f)-C_{i}(f)-P_{i} \subseteq C_{i}(f) .
$$

Hence, $\lambda g_{i, 1}+(1-\lambda) g_{i, 2} \in T_{i}(f)$ and therefore $T_{i}(f)$ is convex. Since $i \in I$ and $f \in K$ are arbitrary, $T_{i}(f)$ is convex, $\forall f \in K$ and $\forall i \in I$.

Hence, our claim is then verified.

Now $\forall i \in I$ and $\forall g_{i} \in K_{i}$; the complement of $T_{i}^{-1}\left(g_{i}\right)$ in $K$ can be defined as

$$
\left[T_{i}^{-1}\left(g_{i}\right)\right]^{c}=\left\{f \in K: F_{i}\left(f, g_{i}\right) \notin-C_{i}(f)\right\} .
$$

From condition (iii) of the above theorem, $\left[T_{i}^{-1}\left(g_{i}\right)\right]^{c}$ will be closed in $K$.

Suppose that $\forall i \in I$ and $\forall f \in K$; we define another setvalued mapping $M_{i}: K \rightarrow 2^{K_{i}}$ by

$$
M_{i}(f)= \begin{cases}A_{i}(f) \cap T_{i}(f) & \text { if } f \in \mathscr{F}_{i} \\ A_{i}(f) ; & \text { if } f \in K \backslash \mathscr{F}_{i} .\end{cases}
$$

Then, it is clear that $\forall i \in I$ and $\forall f \in K, M_{i}(f)$ is convex, because $A(f)$ and $T_{i}(f)$ are both convex. Now, by condition (i), $f_{i} \notin M_{i}(f)$. Since $\forall i \in I$ and $\forall g_{i} \in K_{i}$,

$$
\begin{aligned}
M_{i}^{-1} & \left(g_{i}\right) \\
& =\left(A_{i}^{-1}\left(g_{i}\right) \cap T_{i}^{-1}\left(g_{i}\right)\right) \bigcup\left(\left(K \backslash \mathscr{F}_{i}\right) \cap A_{i}^{-1}\left(g_{i}\right)\right)
\end{aligned}
$$

is open in $K$, because $A_{i}^{-1}\left(g_{i}\right), T_{i}^{-1}\left(g_{i}\right)$ and $K \backslash \mathscr{F}_{i}$ are open in $K$.

Condition (iv) of Theorem 6 is followed from condition (iv). Hence, by fixed point Theorem 6 , there exists $\bar{f} \in K$ such that $M_{i}(\bar{f})=\emptyset, \forall i \in I$. Since $\forall i \in I$ and $\forall f \in K, A_{i}(f)$ is nonempty, we have $A_{i}(\bar{f}) \cap T_{i}(\bar{f})=\emptyset, \forall i \in I$. Therefore, $\forall i \in I, \bar{f}_{i} \in A_{i}(\bar{f})$ and $F_{i}\left(\bar{f}, g_{i}\right) \in-C_{i}(\bar{f}), \forall g_{i} \in A_{i}(\bar{f})$.

This completes the proof.

Now, we establish an existence result for SOQEP (2) involving $\phi$-condensing maps.

Theorem 9. For each $i \in I$, let $K_{i}$ be a nonempty, closed, and convex subset of a locally convex Hausdorff topological vector space $X_{i}$, suppose that $F_{i}: K \times K_{i} \rightarrow Y_{i}$ is a bifunction, and let the set-valued mapping $A=\prod_{i \in I} A_{i}: K \rightarrow 2^{K}$ defined as $A(f)=\prod_{i \in I} A_{i}(f), \forall f \in K$ be $\phi$-condensing. Assume that conditions (i), (ii), and (iii) of Theorem 8 hold. Then SOQEP (2) has a solution.

Proof. In view of Remark 7, it is sufficient to show that the set-valued mapping $S: K \rightarrow 2^{K}$ defined as $S(f)=$ $\prod_{i \in I} S_{i}(f), \forall f \in K$, is $\phi$-condensing, where $S_{i}$ s are the same as defined in the proof of Theorem 8. By the definition of $S_{i}, S_{i}(f) \subseteq A_{i}(f), \forall i \in I$ and $\forall f \in K$ and therefore $S(f) \subseteq$ $A(f), \forall f \in K$. Since $A$ is $\phi$-condensing, by Remark 7, we have $S$ being also $\phi$-condensing.

This completes the proof.

\section{System of Generalized Quasi Operator Equilibrium Problem}

Throughout this section, unless otherwise stated, let $I$ be any index set. For each $i \in I$, let $X_{i}$ be a Hausdorff topological vector space. We denote $L\left(X_{i}, Y_{i}\right)$, the space of all continuous linear operators from $X_{i}$ into $Y_{i}$, where $Y_{i}$ is topological vector space for each $i \in I$ and for each $i \in I$; let $P_{i} \subset Y_{i}$ be a closed, pointed, and convex cone with int $P_{i} \neq \emptyset$, where int $P_{i}$ denotes the interior of set $P_{i}, \forall i \in I$. Consider a family of nonempty convex subsets $\left\{K_{i}\right\}_{i \in I}$ with $K_{i}$ in $L\left(X_{i}, Y_{i}\right)$. Let, for each $i \in I$, a bifunction $F_{i}: K \times K_{i} \rightarrow Y_{i}$ and two set-valued mappings $A_{i}, B_{i}: K \rightarrow 2^{K_{i}}$ be with nonempty values.

Let $e_{i}$ be the unit vector in $Y_{i}$, for each $i \in I$, and also $\alpha_{i} e_{i}, \beta_{i} e_{i} \in Y_{i}$ such that $\alpha_{i} e_{i} \nless_{P_{i}} \beta_{i} e_{i}$, where $\alpha_{i}, \beta_{i} \in \mathbb{R}$ are two real numbers such that $\alpha_{i} \leq \beta_{i}$.

Now, we consider the system of generalized quasi operator equilibrium problems (for short, SGQOEP). Find $\bar{f} \in K$ such that, for each $i \in I$,

$$
\bar{f}_{i} \in B_{i}(\bar{f}), \quad \alpha_{i} e_{i} \nless_{P_{i}} F_{i}\left(\bar{f}, g_{i}\right){ }_{P_{i}} \beta_{i} e_{i} ; \quad \forall g_{i} \in A_{i}(\bar{f}) .
$$

\subsection{Special Cases}

(I) If $A_{i}=B_{i}, \forall i$, then SGQOEP (10) reduces to finding of $\bar{f} \in K$ such that, for each $i \in I$,

$$
\alpha_{i} e_{i} \nless_{P} F_{i}\left(\bar{f}, g_{i}\right) \nless_{P} \beta_{i} e_{i} ; \quad \forall g_{i} \in A_{i}(\bar{f}) .
$$


(II) If, in Case (I), we take $Y_{i}=\mathbb{R}$, then $P_{i}=\mathbb{R}_{+}$and $e_{i}=1$; then problem (10) reduces to the system of generalized quasi operator equilibrium problems with lower and upper bounds (for short, SGQOEPLUB). Find $\bar{f} \in K$ such that, for each $i \in I$,

$$
\bar{f}_{i} \in B_{i}(\bar{f}), \quad \alpha_{i} \leq F_{i}\left(\bar{f}, g_{i}\right) \leq \beta_{i} ; \quad \forall g_{i} \in A_{i}(\bar{f})
$$

Now, we establish the existence result for SGQOEP (10).

Theorem 10. For each $i \in I$, let $K_{i}$ be a nonempty convex subset of a topological vector space $X_{i}$ and $F_{i}, P_{i}, Q_{i}: K \times K_{i} \rightarrow$ $K_{i}$ are the bifunctions, $B_{i}: K \rightarrow 2^{K_{i}}$ is a set-valued mapping such that the set $F_{i}=\left\{f \in K: f_{i} \in B_{i}(f)\right\}$ is compactly closed, $A_{i}: K \rightarrow 2^{K_{i}}$ is a set-valued mapping with nonempty values such that, for each $g_{i} \in K_{i}, A_{i}^{-1}\left(g_{i}\right)$ is compactly open in $K$, and $\forall i \in I, e_{i} \in Y_{i}$ are the unit vector such that $\alpha_{i} e_{i} \nless_{P_{i}} \beta_{i} e_{i}$, where $\alpha_{i}, \beta_{i} \in \mathbb{R}$ are two real numbers such that $\alpha_{i} \leq \beta_{i}$. For each $i \in I$, assume that the following conditions hold:

(i) for all $f \in K, \operatorname{CoA}_{i}(f) \subseteq B_{i}(f)$;

(ii) for all $f \in K, \alpha_{i} e_{i}>_{P_{i}} P_{i}\left(f, f_{i}\right)$ or $Q_{i}\left(f, f_{i}\right)>_{P_{i}} \beta_{i} e_{i}$;

(iii) for all $f \in K$ and for every nonempty finite subset $N_{i} \subseteq$ $\left\{g_{i} \in K_{i}: F_{i}\left(f, g_{i}\right)<_{P_{i}} \alpha_{i} e_{i}\right.$ or $\left.F_{i}\left(f, g_{i}\right)>_{P_{i}} \beta_{i} e_{i}\right\}$, we have

$\operatorname{CoN}_{i} \subseteq\left\{g_{i} \in K_{i}: P_{i}\left(f, g_{i}\right) \nless_{P_{i}} \alpha_{i} e_{i}\right.$ and $\left.Q_{i}\left(f, g_{i}\right) \ngtr_{P_{i}} \beta_{i} e_{i}\right\}$;

(iv) for all $g_{i} \in K_{i}$, the set $\left\{f \in K: \beta_{i} e_{i} \nless_{P_{i}} F_{i}\left(f, g_{i}\right) \nless_{P_{i}} \alpha_{i} e_{i}\right.$ is compactly closed in $K$;

(v) there exist a nonempty compact subset $D$ of $K$ and a nonempty compact convex subset $E_{i} \subset K_{i}$, for each $i \epsilon$ $I$, such that, for all $f \in K \backslash D$, there exists $i \in I$ such that $\widehat{g}_{i} \in E_{i}$ satisfying $\widehat{g}_{i} \in A_{i}(f)$ and either $F_{i}\left(f, \widehat{g}_{i}\right)_{P_{i}}<$ $\alpha_{i} e_{i}$ or $F_{i}\left(f, \widehat{g}_{i}\right)>_{P_{i}} \beta_{i} e_{i}$.

Then the problem SGQOEP (10) has a solution.

Proof. For each $i \in I$ and for all $f \in K$, define two set-valued mappings $G_{i}, H_{i}: K \rightarrow 2^{K_{i}}$ by

$$
\begin{gathered}
G_{i}(f)=\left\{g_{i} \in K_{i}: F_{i}\left(f, g_{i}\right)<{ }_{P_{i}} \alpha_{i} e_{i} \text { or } F_{i}\left(f, g_{i}\right)>_{P_{i}} \beta_{i} e_{i}\right\}, \\
H_{i}(f)=\left\{g_{i} \in K_{i}: P_{i}\left(f, g_{i}\right)_{P_{i}} \nless \alpha_{i} e_{i} \text { and } Q_{i}\left(f, g_{i}\right) \ngtr_{P_{i}} \beta_{i} e_{i}\right\} .
\end{gathered}
$$

Condition (iii) implies that, for each $i \in I$ and for all $f \in K$, $\operatorname{CoG}_{i}(f) \subseteq H_{i}(f)$.

From condition (ii), we have $f_{i} \notin H_{i}(f)$ for all $f \in K$ and for each $i \in I$.

Thus, for each $i \in I$ and for all $g_{i} \in K_{i}$,

$$
G_{i}^{-1}\left(g_{i}\right)=\left\{f \in K: F_{i}\left(f, g_{i}\right)<{ }_{P_{i}} \alpha_{i} e_{i} \text { or } F_{i}\left(f, g_{i}\right)>_{P_{i}} \beta_{i} e_{i}\right\} \text {. }
$$

We have complement of $G_{i}^{-1}\left(g_{i}\right)$ in $K$ :

$$
\left[G_{i}^{-1}\left(g_{i}\right)\right]^{c}=\left\{\beta_{i} e_{i} \nless_{P_{i}} F_{i}\left(\bar{f}, g_{i}\right) \nless_{P_{i}} \alpha_{i} e_{i}\right\},
$$

which is compactly closed by virtue of condition (iv). Therefore, for each $i \in I$ and for all $g_{i} \in K_{i}, G_{i}^{-1}\left(g_{i}\right)$ is compactly open in $K$.

For each $i \in I$, define two set-valued mappings $S_{i}, T_{i}$ : $K \rightarrow 2^{K_{i}}$ by

$$
\begin{aligned}
& S_{i}(f)= \begin{cases}G_{i}(f) \cap A_{i}(f) ; & \text { if } f \in \mathscr{F}_{i} \\
A_{i}(f) ; & \text { if } f \in K \backslash \mathscr{F}_{i},\end{cases} \\
& T_{i}(f)= \begin{cases}H_{i}(f) \cap B_{i}(f) ; & \text { if } f \in \mathscr{F}_{i} \\
B_{i}(f) ; & \text { if } f \in K \backslash \mathscr{F}_{i} .\end{cases}
\end{aligned}
$$

Thus, for each $i \in I$ and for all $f \in K, \operatorname{CoG}_{i}(f) \subseteq H_{i}(f)$ and in view of condition (i), we obtain $\operatorname{CoS}_{i}(f) \subseteq T_{i}(f)$. It is easy to see that

$$
S_{i}^{-1}\left(g_{i}\right)=\left(A_{i}^{-1}\left(g_{i}\right) \cap G_{i}^{-1}\left(g_{i}\right)\right) \bigcup\left(\left(K \backslash \mathscr{F}_{i}\right) \cap A_{i}^{-1}\left(g_{i}\right)\right)
$$

for each $i \in I$ and for all $f \in K$. Thus, for each $i \in I$ and for all $G_{i}{ }^{-1}\left(g_{i}\right), A_{i}{ }^{-1}\left(g_{i}\right)$ and $K \backslash \mathscr{F}_{i}$ are compactly open in $K$. We have $S_{i}^{-1}\left(g_{i}\right)$ being compactly open in $K$. Also $f_{i} \notin T_{i}(f)$ for all $f \in K$ and for each $i \in I$.

Then, by Theorem 5 , there exists $\bar{f} \in K$ such that $S_{i}(\bar{f})=$ $\emptyset$ for each $i \in I$. If $\bar{f} \in K \backslash \mathscr{F}_{i}$, then $A_{i}(\bar{f})=S_{i}(\bar{f})=\emptyset$, which contradicts the fact that $A_{i}(f)$ is nonempty for each $i \in I$ and for all $f \in X$. Hence, $\bar{f} \in \mathscr{F}_{i}$, for each $i \in I$. Therefore, $\bar{f}_{i} \in B_{i}(\bar{f})$ and $G_{i}(\bar{f}) \cap A_{i}(\bar{f})=\emptyset$, for all $i \in I$. Thus, for each $i \in I, \bar{f}_{i} \in B_{i}(\bar{f})$ and $\beta_{i} e_{i} \nless_{P_{i}} F_{i}\left(\bar{f}, g_{i}\right) \nless_{P_{i}} \alpha_{i} e_{i}$ for all $g_{i} \in A_{i}(\bar{f})$. This completes the proof.

Now, we establish an existence result for SGQOEP (10) involving $\phi$-condensing maps.

Theorem 11. For each $i \in I$, assume that conditions (i)-(iv) of Theorem 10. hold. Let $\phi$ be a measure of noncompactness on $\prod_{i \in I} X_{i}$. Further, assume that the set-valued mapping $B: K \rightarrow$ $2^{K}$ defined as $K_{i}$ is a nonempty, closed, and convex subset of a locally convex Hausdorff topological vector space $X_{i}$ and $F_{i}$ : $K \times K_{i} \rightarrow Y_{i}$ is a bifunction and let the set-valued mapping $A=$ $\prod_{i \in I} A_{i}: K \rightarrow 2^{K}$ defined as $B(f)=\prod_{i \in I} B_{i}(f), \forall f \in K$ be $\phi$-condensing. Then, there exists a solution $\bar{f} \in K$ of SGQOEP (10).

Proof. In view of Remark 7, it is sufficient to show that the set-valued mapping $T: K \rightarrow 2^{K}$ defined as $T(f)=$ $\prod_{i \in I} T_{i}(f), \forall f \in K$, is $\phi$-condensing, where $T_{i}$ s are the same as defined in the proof of Theorem 10. By the definition of $T_{i}, T_{i}(f) \subseteq B_{i}(f), \forall i \in I$ and $\forall f \in K$ and therefore $S(f) \subseteq$ $A(f), \forall f \in K$. Since $B$ is $\phi$-condensing, by Remark 7, we have $T$ being also $\phi$-condensing.

This completes the proof.

Next, we derive the existence result for the solution of SGQOEPLUB (12). 
Corollary 12. For each $i \in I$, let $K_{i}$ be a nonempty convex subset of a topological vector space $X_{i}$ and $F_{i}, L_{i}, Q_{i}: K \times K_{i} \rightarrow$ $\mathbb{R}$ are the bifunctions, $B_{i}: K \rightarrow 2^{K_{i}}$ is a set-valued mapping such that the set $\mathscr{F}_{i}=\left\{f \in K: f_{i} \in B_{i}(f)\right\}$ is compactly closed, $A_{i}: K \rightarrow 2^{K_{i}}$ is a set-valued mapping with nonempty values such that, for each $g_{i} \in K_{i}, A_{i}{ }^{-1}\left(g_{i}\right)$ is compactly open in $K$, and $\alpha_{i}, \beta_{i} \in \mathbb{R}$ are two real numbers such that $\alpha_{i} \leq \beta_{i}$. For each $i \in I$, assume that the following conditions hold:

(i) for all $f \in K, \operatorname{Co}_{i}(f) \subseteq B_{i}(f)$;

(ii) for all $f \in K, L_{i}\left(f, f_{i}\right)<\alpha_{i}$ or $Q_{i}\left(f, f_{i}\right)>\beta_{i}$;

(iii) for all $f \in K$ and for every nonempty finite subset $N_{i} \subseteq$ $\left\{g_{i} \in K_{i}: F_{i}\left(f, g_{i}\right)<\alpha_{i}\right.$ or $\left.F_{i}\left(f, g_{i}\right)>\beta_{i}\right\}$, we have

$\mathrm{CoN}_{i} \subseteq\left\{g_{i} \in K_{i}: L_{i}\left(f, g_{i}\right) \geq \alpha_{i}\right.$ and $\left.Q_{i}\left(f, g_{i}\right) \leq \beta_{i}\right\} ;$

(iv) for all $g_{i} \in K_{i}$, the set $\left\{f \in K: \alpha_{i} \leq F_{i}\left(f, g_{i}\right) \leq \beta_{i}\right\}$ is compactly closed in $K$;

(v) there exist a nonempty compact subset $D$ of $K$ and a nonempty compact convex subset $E_{i} \subset K_{i}$, for each $i \epsilon$ $I$, such that, for all $f \in K \backslash D$, there exists $i \in I$ such that $\widehat{g}_{i} \in E_{i}$ satisfying $\widehat{g}_{i} \in A_{i}(f)$ and either $F_{i}\left(f, \widehat{g}_{i}\right)<\alpha_{i}$ or $F_{i}\left(f, \hat{g}_{i}\right)>\beta_{i}$.

Then the problem SGQOEPLUB (12) has a solution.

Proof. For each $i \in I$ and for all $f \in K$, define two set-valued mappings $G_{i}, H_{i}: K \rightarrow 2^{K_{i}}$ by

$$
\begin{gathered}
G_{i}(f)=\left\{g_{i} \in K_{i}: F_{i}\left(f, g_{i}\right)<\alpha_{i} \text { or } F_{i}\left(f, g_{i}\right)>\beta_{i}\right\}, \\
H_{i}(f)=\left\{g_{i} \in K_{i}: L_{i}\left(f, g_{i}\right) \geq \alpha_{i} \text { and } Q_{i}\left(f, g_{i}\right) \leq \beta_{i}\right\} .
\end{gathered}
$$

Condition (iii) implies that, for each $i \in I$ and for all $f \in K$, $\mathrm{CoG}_{i}(f) \subseteq H_{i}(f)$.

From condition (ii), we have $f_{i} \notin H_{i}(f)$ for all $f \in K$ and for each $i \in I$.

Thus, for each $i \in I$ and for all $g_{i} \in K_{i}$,

$$
G_{i}^{-1}\left(g_{i}\right)=\left\{f \in K: F_{i}\left(f, g_{i}\right)<\alpha_{i} \text { or } F_{i}\left(f, g_{i}\right)>\beta_{i}\right\} .
$$

We have complement of $G_{i}^{-1}\left(g_{i}\right)$ in $K$ :

$$
\left[G_{i}^{-1}\left(g_{i}\right)\right]^{c}=\left\{\alpha_{i} \leq F_{i}\left(f, g_{i}\right) \leq \beta_{i}\right\},
$$

which is compactly closed by virtue of condition (iv). Therefore, for each $i \in I$ and for all $g_{i} \in K_{i}, G_{i}^{-1}\left(g_{i}\right)$ is compactly open in $K$.

For each $i \in I$, define two set-valued mappings $S_{i}, T_{i}$ : $K \rightarrow 2_{i}^{K}$ by

$$
\begin{aligned}
& S_{i}(f)= \begin{cases}G_{i}(f) \cap A_{i}(f) & \text { if } f \in \mathscr{F}_{i} \\
A_{i}(f) ; & \text { if } f \in K \backslash \mathscr{F}_{i},\end{cases} \\
& T_{i}(f)= \begin{cases}H_{i}(f) \cap B_{i}(f) & \text { if } f \in \mathscr{F}_{i} \\
B_{i}(f) ; & \text { if } f \in K \backslash \mathscr{F}_{i} .\end{cases}
\end{aligned}
$$

Thus, for each $i \in I$ and for all $f \in K, \operatorname{CoG}_{i}(f) \subseteq H_{i}(f)$ and in view of condition (i), we obtain $\operatorname{CoS}_{i}(f) \subseteq T_{i}(f)$. It is easy to see that

$$
S_{i}^{-1}\left(g_{i}\right)=\left(A_{i}^{-1}\left(g_{i}\right) \cap G_{i}^{-1}\left(g_{i}\right)\right) \bigcup\left(\left(K \backslash \mathscr{F}_{i}\right) \cap A_{i}^{-1}\left(g_{i}\right)\right)
$$

for each $i \in I$ and for all $f \in K$. Thus, for each $i \in I$ and for all $G_{i}{ }^{-1}\left(g_{i}\right), A_{i}{ }^{-1}\left(g_{i}\right)$ and $K \backslash \mathscr{F}_{i}$ are compactly open in $K$. We have $S_{i}^{-1}\left(g_{i}\right)$ being compactly open in $K$. Also $f_{i} \notin T_{i}(f)$ for all $f \in K$ and for each $i \in I$.

Then, by Theorem 5 , there exists $\bar{f} \in K$ such that $S_{i}(\bar{f})=$ $\emptyset$ for each $i \in I$. If $\bar{f} \in K \backslash \mathscr{F}_{i}$, then $A_{i}(\bar{f})=S_{i}(\bar{f})=\emptyset$, which contradicts the fact that $A_{i}(f)$ is nonempty for each $i \in I$ and for all $f \in X$. Hence, $\bar{f} \in \mathscr{F}_{i}$, for each $i \in I$. Therefore, $\bar{f}_{i} \in B_{i}(\bar{f})$ and $G_{i}(\bar{f}) \cap A_{i}(\bar{f})=\emptyset$, for all $i \in I$. Thus, for each $i \in I, \bar{f}_{i} \in B_{i}(\bar{f})$ and $\alpha_{i} \leq F_{i}\left(\bar{f}, g_{i}\right) \leq \beta_{i}$ for all $g_{i} \in A_{i}(\bar{f})$. This completes the proof.

Now, we establish an existence result for SGQOEPLUB (12) involving $\phi$-condensing maps.

Theorem 13. For each $i \in I$, assume that conditions (i)-(iv) of Corollary 12 hold. Let $\phi$ be a measure of noncompactness on $\prod_{i \in I} X_{i}$. Further, assume that the set-valued mapping $B$ : $K \rightarrow 2^{K}$ defined as $K_{i}$ is a nonempty, closed, and convex subset of a locally convex Hausdorff topological vector space $X_{i}$ and $F_{i}: K \times K_{i} \rightarrow \mathbb{R}$ is a bifunction and let the set-valued mapping $A=\prod_{i \in I} A_{i}: K \rightarrow 2^{K}$ defined as $B(f)=\prod_{i \in I} B_{i}(f), \forall f \in$ $K$, be $\phi$-condensing. Then, there exists a solution $\bar{f} \in K$ of SGQOEPLUB (12).

Proof. In view of Remark 7, it is sufficient to show that the set-valued mapping $T: K \rightarrow 2^{K}$ defined as $T(f)=$ $\prod_{i \in I} T_{i}(f), \forall f \in K$, is $\phi$-condensing, where $T^{i} s$ are the same as defined in the proof of Theorem 10. By the definition of $T_{i}, T_{i}(f) \subseteq B_{i}(f), \forall i \in I$ and $\forall f \in K$ and therefore $S(f) \subseteq$ $A(f), \forall f \in K$. Since $B$ is $\phi$-condensing, by Remark 7, we have $T$ being also $\phi$-condensing.

This completes the proof.

\section{Conflict of Interests}

The author declares that there is no conflict of interests regarding the publication of this paper.

\section{References}

[1] A. Domokos and J. Kolumbán, "Variational inequalities with operator solutions," Journal of Global Optimization, vol. 23, no. 1, pp. 99-110, 2002.

[2] K. R. Kazmi and A. Raouf, "A class of operator equilibrium problems," Journal of Mathematical Analysis and Applications, vol. 308, no. 2, pp. 554-564, 2005.

[3] Q. H. Ansari, W. K. Chan, and X. Q. Yang, "The system of vector quasi-equilibrium problems with applications," Journal of Global Optimization, vol. 29, no. 1, pp. 45-57, 2004. 
[4] Q. H. Ansari, S. Schaible, and J. C. Yao, "System of vector equilibrium problems and its applications," Journal of Optimization Theory and Applications, vol. 107, no. 3, pp. 547-557, 2000.

[5] P. Deguire, K. K. Tan, and G. X.-Z. Yuan, "The study of maximal elements, fixed points for $L_{S}$-majorized mappings and their applications to minimax and variational inequalities in product topological spaces," Nonlinear Analysis: Theory, Methods \& Applications, vol. 37, no. 7, pp. 933-951, 1999.

[6] S. Al-Homidan and Q. H. Ansari, "Systems of quasi-equilibrium problems with lower and upper bounds," Applied Mathematics Letters, vol. 20, no. 3, pp. 323-328, 2007.

[7] P. M. Fitzpatrick and W. V. Petryshyn, "Fixed point theorems for multivalued noncompact acyclic mappings," Pacific Journal of Mathematics, vol. 54, no. 2, pp. 17-23, 1974.

[8] L.-J. Lin and Q. H. Ansari, "Collective fixed points and maximal elements with applications to abstract economies," Journal of Mathematical Analysis and Applications, vol. 296, no. 2, pp. 455472, 2004. 


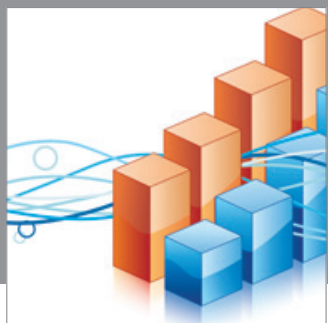

Advances in

Operations Research

mansans

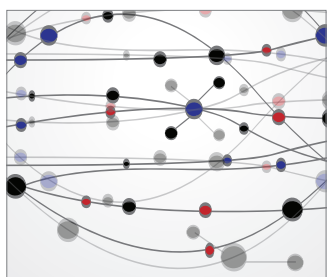

The Scientific World Journal
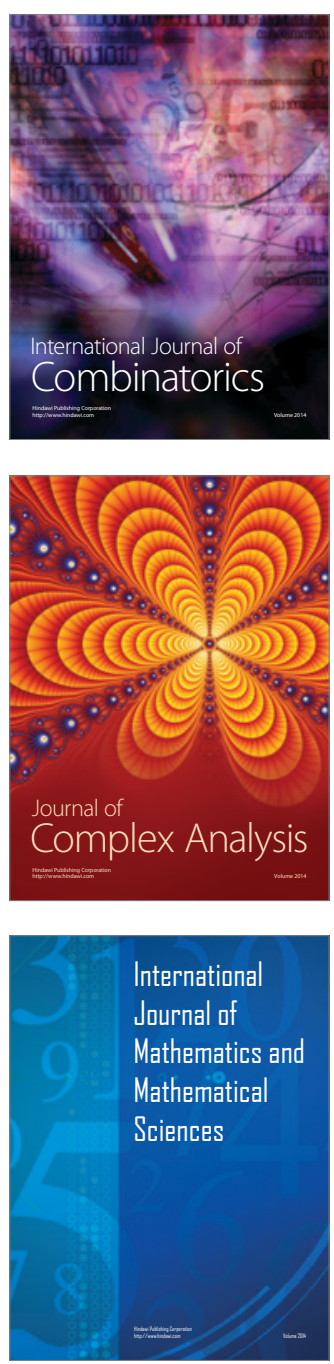
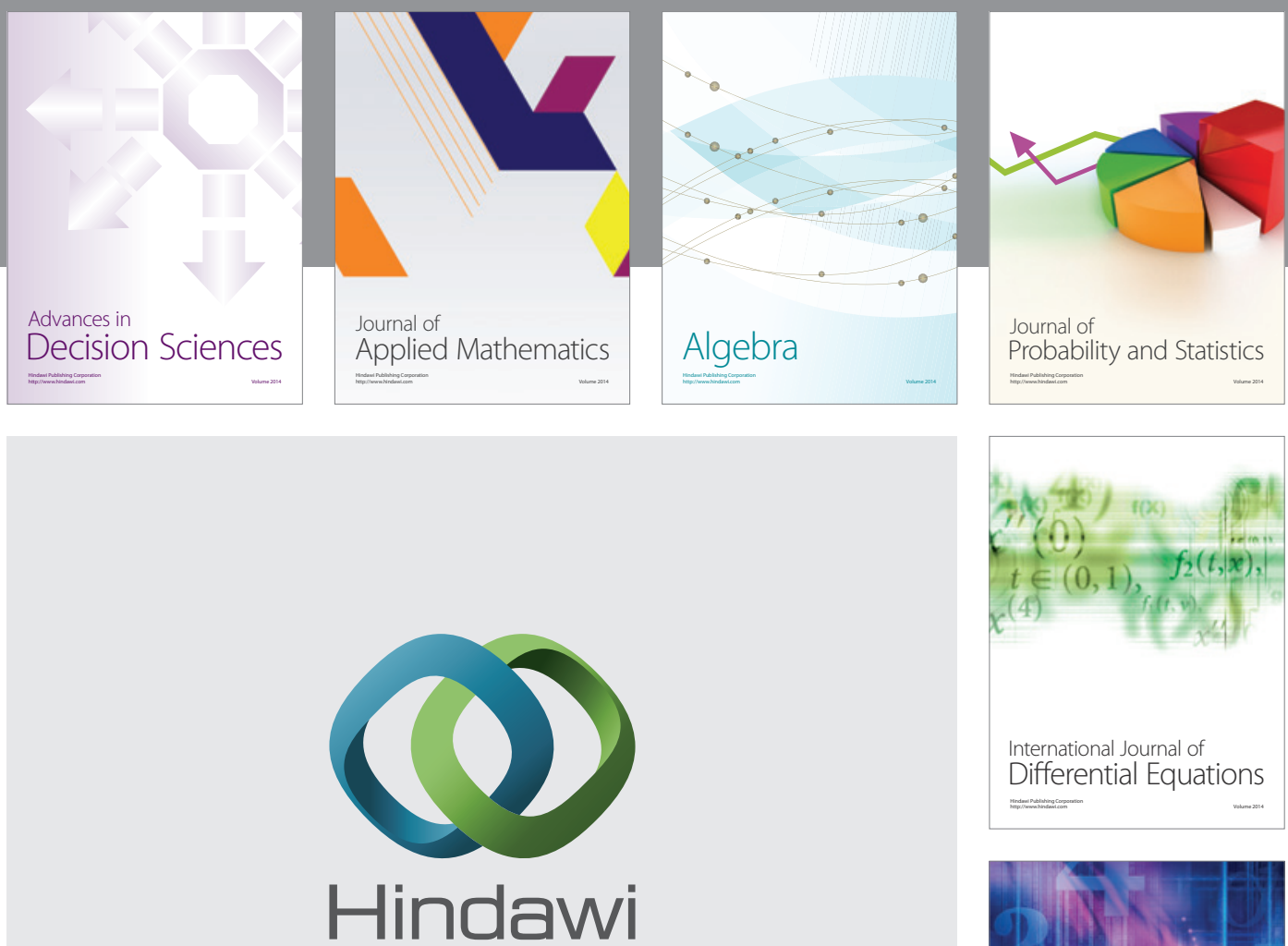

Submit your manuscripts at http://www.hindawi.com
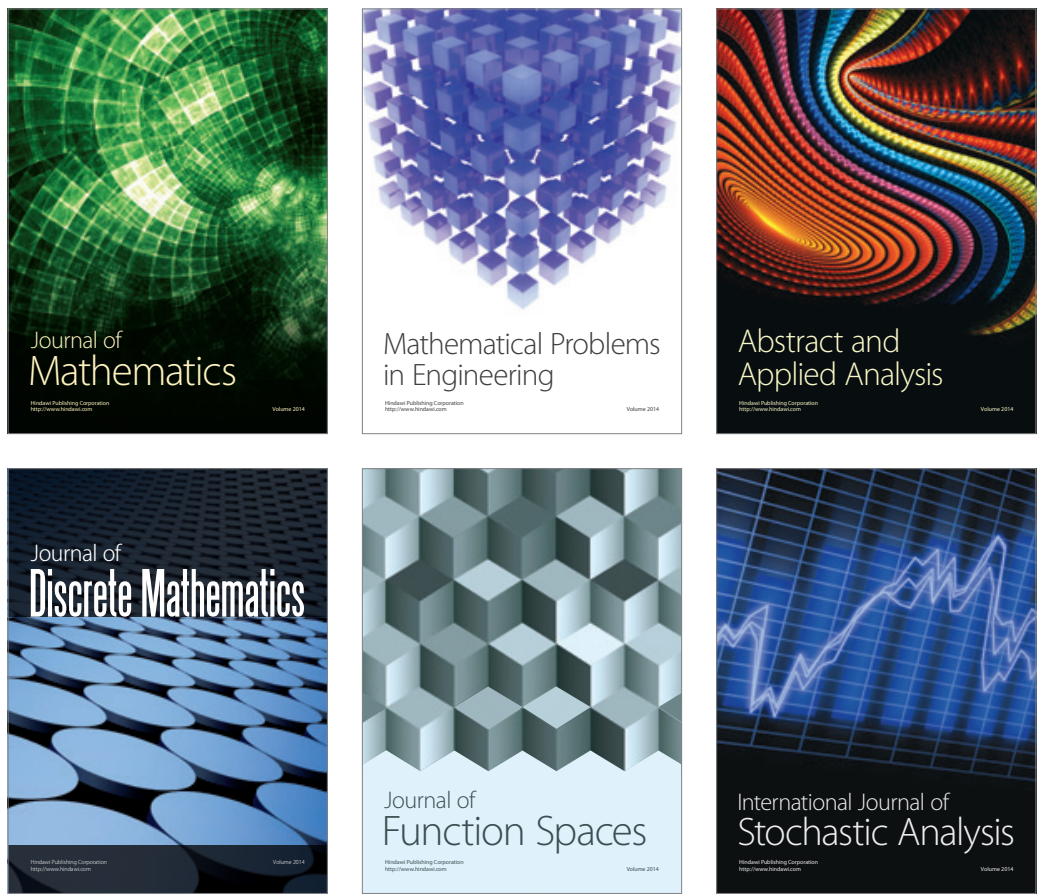

Journal of

Function Spaces

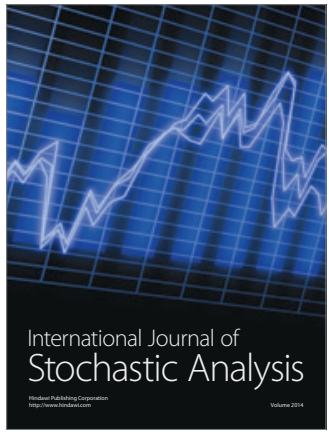

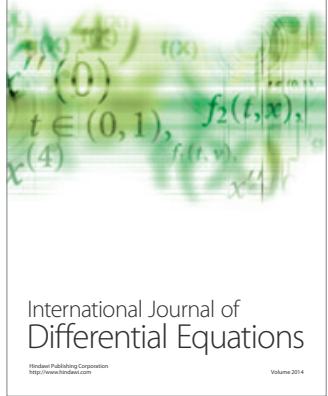
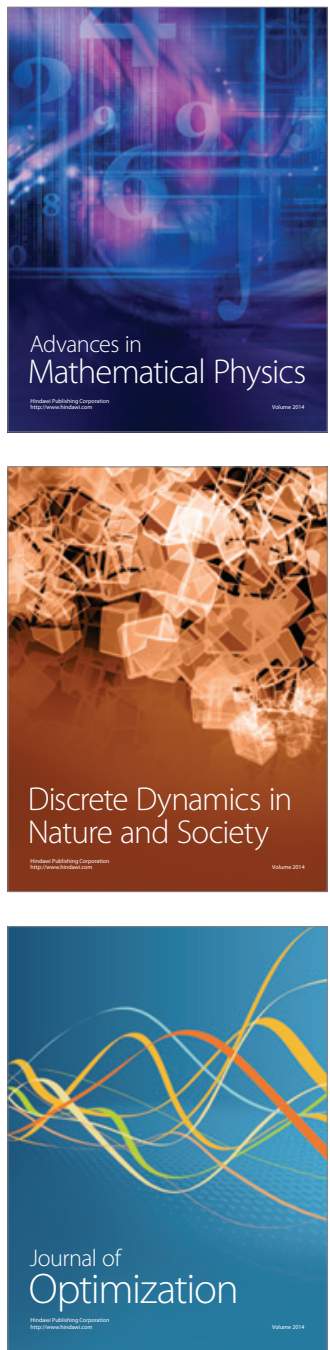